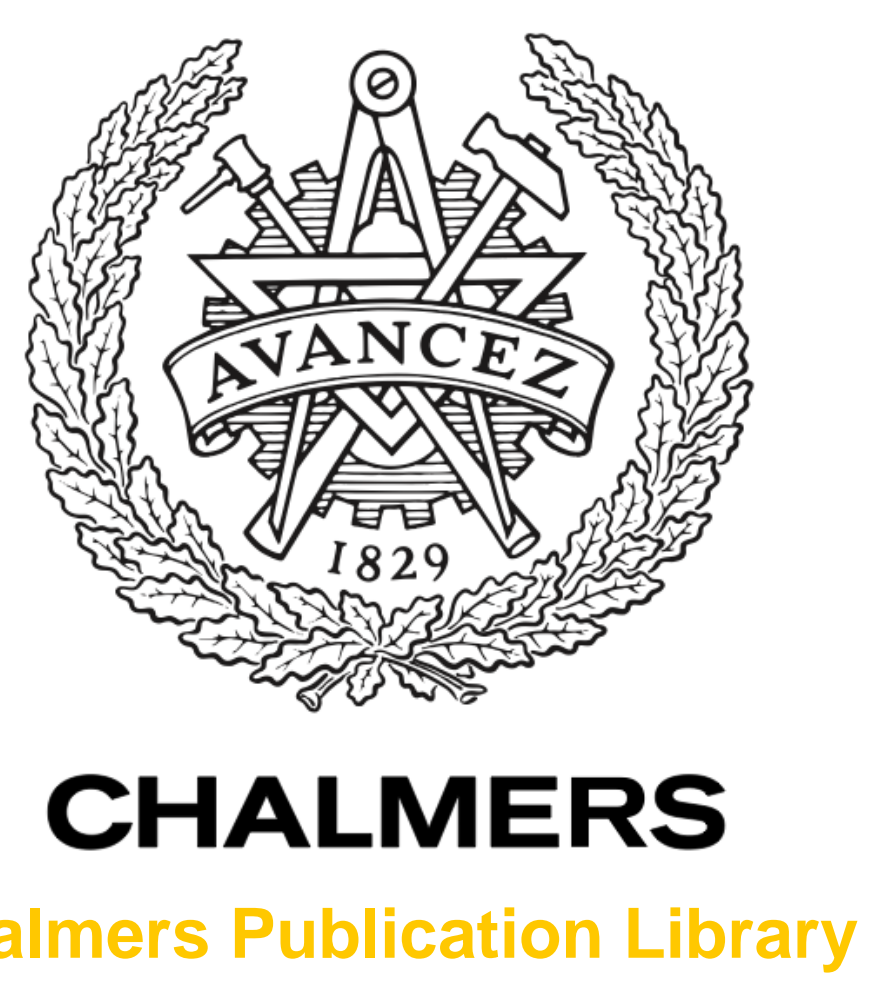

Chalmers Publication Library

\title{
Distributed Serially Concatenated Codes for Multi-Source Cooperative Relay Networks
}

This document has been downloaded from Chalmers Publication Library (CPL). It is the author's version of a work that was accepted for publication in:

IEEE Transactions on Wireless Communications (ISSN: 1536-1276)

Citation for the published paper:

Youssef, R. ; Graell i Amat, A. (2011) "Distributed Serially Concatenated Codes for Multi-

Source Cooperative Relay Networks". IEEE Transactions on Wireless Communications, vol.

10(1), pp. 253-263.

http://dx.doi.org/10.1109/TWC.2010.102810.10042

Down「oaded from: http://publications.lib.chalmers.se/publication/156953

Notice: Changes introduced as a result of publishing processes such as copy-editing and formatting may not be reflected in this document. For a definitive version of this work, please refer to the published source. Please note that access to the published version might require a subscription.

Chalmers Publication Library (CPL) offers the possibility of retrieving research publications produced at Chalmers University of Technology. It covers all types of publications: articles, dissertations, licentiate theses, masters theses, conference papers, reports etc. Since 2006 it is the official tool for Chalmers official publication statistics. To ensure that Chalmers research results are disseminated as widely as possible, an Open Access Policy has been adopted.

The CPL service is administrated and maintained by Chalmers Library. 


\title{
Distributed Serially Concatenated Codes for Multi-Source Cooperative Relay Networks
}

\author{
Roua Youssef, Student Member, IEEE, and Alexandre Graell i Amat, Senior Member, IEEE
}

\begin{abstract}
In this paper, we propose a distributed turbo-like coding scheme for a multi-source relay scenario where multiple sources communicate with a destination with the help of a common relay, which uses the decode-and-forward strategy and operates in half-duplex mode. The proposed distributed code can be viewed as a serially concatenated code (SCC). Thus, at the destination decoding is performed in an iterative fashion which resembles the decoding of a classic SCC. We consider the two scenarios where the sources transmit over orthogonal channels and where they do not. For the latter, interleave-division multipleaccess is used for multiuser detection. For both scenarios we optimize the transmission time allocated to the sources and to the relay and compute the achievable rates. The proposed scheme achieves very low error rates and offers significant performance gains with respect to non-cooperation, even for a very large number of sources. Furthermore, it provides a high flexibility in terms of code rate, number of sources, overall system rate and error protection.
\end{abstract}

\section{INTRODUCTION}

In the last years, we have witnessed an increasing interest in the research community on cooperation techniques for wireless communication networks. The key idea of cooperative communication is that, exploiting the inherent broadcast nature of the wireless channel, several nodes help each other to transmit information to the destination. Cooperative communications bring higher data throughput, spectral and power efficiency, and reliability.

User cooperation was first discussed in the pioneering paper by van der Meulen on the relay channel [1]. The relay channel was further investigated by Cover and El Gamal in [2], where upper and lower bounds to the capacity where derived, and two basic coding strategies were proposed: decode-andforward, and compress-and-forward, where the relay decodes and compresses, respectively, the source information prior to forwarding. Decode-and-forward strategy is relevant for the scenario where the relay is close to the source, while compress-and-forward is the optimal strategy when the relay is close to the destination.

In order to harvest the gains predicted by information theory, practical cooperation schemes have been recently proposed in $[3,4]$. In [3], a novel coding technique for the relay channel

R. Youssef and A. Graell i Amat are with the Department of Electronics, Institut TELECOM-TELECOM Bretagne, CS 83818 29238 Brest Cedex 3, France (e-mail: roua.youssef@telecom-bretagne.eu, alexandre.graell@telecom-bretagne.eu).

This work was partially funded by a Marie Curie Intra-European Fellowship within the 6th European Community Framework Programme, by the Pôle de Recherche Avancée en Communications (PRACOM) and by NEWCOM++.

The material in this paper was presented in part at the 2010 IEEE International Conference on Communications, Cape Town, South Africa, May 2010 . called distributed turbo code was proposed. In the proposed scheme, the source broadcasts to both the relay and the destination. The relay decodes, interleaves and re-encodes the message prior to forwarding. The destination receives two encoded copies of the original message and jointly decodes them by an iterative decoding algorithm. The proposed technique achieves a combined diversity and coding gain. More recently, joint network and channel coding schemes have been proposed in [5,6] for a multiple-access relay channel. In [5], two sources transmit to both the destination and the relay, which forwards a combination (a simple XOR) of the received messages from both sources. In these works, the number of sources is limited to at most two, and the extension to multiple sources with good performance is not straightforward.

In this paper, we propose a distributed serially concatenated coding scheme for a more general multi-source wireless relay network where multiple sources transmit to a single destination with the help of a common relay, which uses the decodeand-forward strategy and operates in half-duplex mode. Halfduplex relaying is relevant from practical purposes, since it is considered a challenge to provide full duplex operation at the relay [7, p. 324]. The relay decodes the received noisy observations from the sources, and properly combines and re-encodes the estimated codewords to generate some extra redundancy. The amount of redundancy generated by the relay can easily be tuned according to performance requirements and/or constraints in terms of throughput and power. The overall communication network can be viewed as a serially concatenated code (SCC), where the outer encoder groups the encoders of the sources and the inner encoder is the relay. Accordingly, the receiver can decode sources data using a decoding strategy that resembles the decoding of a SCC.

We consider the two scenarios where the sources transmit over orthogonal channels and where they do not. For the latter, interleave-division multiple access (IDMA) is used for multiuser detection. For both scenarios we compute the achievable rates. Furthermore, for the orthogonal case we investigate the optimal allocation of resources between the relay and the sources.

The proposed scheme achieves very low error rates and offers significant performance gains with respect to noncooperation, even for a very large number of sources. A typical scenario for the proposed coding scheme is a wireless sensor network where several sensors process small amounts of information and communicate to a destination with the help of a central node, which has less stringent restrictions on resources.

A similar scenario has been considered in [8], where each source encodes its own information using the same block 


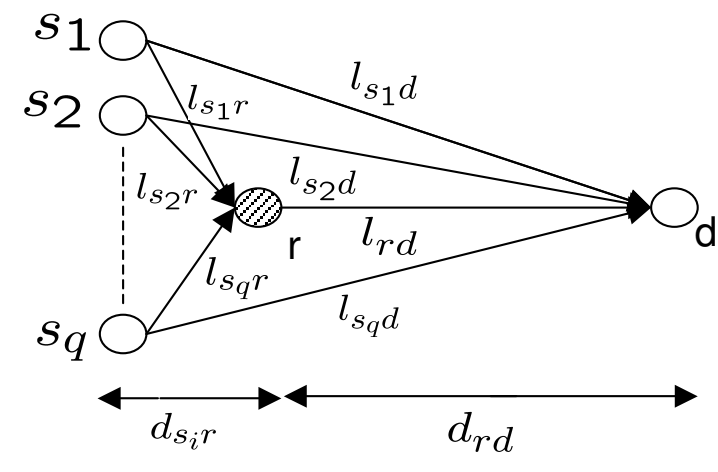

Fig. 1. A wireless relay network: multiple sources transmit to a single destination with the help of a common relay.

code. The relay decodes the codewords from the sources, places them in a matrix and then re-encodes the columns using another linear block code. The overall scheme can be regarded as a turbo product code. Significant performance gains with respect to the non-cooperation case are achieved. However, the scheme in [8] presents several drawbacks. First, it lacks in flexibility. For instance, changing the number of sources requires the use of a different block code at the relay. Our proposed scheme provides a higher flexibility in terms of code rate, number of sources, throughput and error protection level with respect to the approach in [8].

The remainder of this paper is organized as follows. The system model is defined in Section II. In Section III, we introduce two different encoding strategies at the relay and discuss code design. We further present the overall network as a SCC in Section IV. Section V discusses some informationtheoretic limits in terms of achievable rates. Convergence properties of the proposed distributed SCC are addressed in Section VI by means of an extrinsic information transfer chart analysis. Section VII presents the proposed scheme in a non-orthogonal scenario where IDMA is used for multiuser detection. Finally, numerical results are given in Section VIII and some conclusions are drawn in Section IX.

\section{System ModeL}

We consider the wireless relay network depicted in Fig. 1. The network consists of $q$ sources, $\mathrm{s}_{1}, \ldots, \mathrm{s}_{q}$, which communicate statistically independent data to a single destination $d$ cooperating through a common relay $r$, which uses the decode-and-forward strategy and operates in half-duplex mode according to a time-division transmission schedule.

\section{A. Orthogonal channels}

The transmission time is divided into $q+1$ time slots. At source $\mathrm{s}_{i}(i=1, \ldots, q)$ the information sequence $\mathbf{u}_{\mathrm{s}_{i}}$, of length $k_{i}$ bits, is encoded by encoder $\mathcal{C}_{i}$ of rate $R_{i}$ into codeword $\mathbf{x}_{\mathrm{s}_{i}}$ of length $n_{i}=k_{i} / R_{i}$ bits, which is transmitted over the wireless channel at time slot $i$. Here, we consider very simple rate- $1 / 2,4$-state convolutional encoders (possibly punctured to higher rates) for $\mathcal{C}_{i}$. Stronger codes, such as turbo codes, can also be used, at the expense of a more complex decoder at the destination. Furthermore, for the scenario considered in this paper (relay is closer to the sources than to the destination) the use of stand-alone codes at the sources leads to better convergence compared to iteratively decodable codes, while good error floors are yet achieved.

Due to the broadcast nature of the wireless channel the relay receives a noisy observation of codewords $\mathbf{x}_{\mathrm{s}_{i}}$, denoted by $\mathbf{y}_{\mathrm{s}_{i} \mathrm{r}}$, from the different sources. It then cooperates with them by transmitting its own parity sequence $\mathbf{x}_{\mathrm{r}}$ to the destination at time slot $q+1$. The destination decodes the information of the $q$ sources by jointly exploiting the received sequences $\mathbf{y}_{\mathbf{s}_{i}} \mathrm{~d}$ from the sources and the sequence $\mathbf{y}_{\text {rd }}$ from the relay.

As shown in Fig. 1, there are three directed transmission links under consideration: the links from the sources to the destination, $l_{\mathrm{s}_{i} \mathrm{~d}}$, the links from the sources to the relay, $l_{\mathrm{s}_{i} \mathrm{r}}$, and the link from the relay to the destination, $l_{\mathrm{rd}}$. We denote by $\gamma_{\mathrm{s}_{i} \mathrm{~d}}, \gamma_{\mathrm{s}_{i} \mathrm{r}}$ and $\gamma_{\mathrm{rd}}$ the signal-to-noise ratio (SNR) of the $i$-th source-to-destination channel, the $i$-th source-to-relay channel, and the relay-to-destination channel, respectively. Likewise, we denote by $d_{\mathrm{s}_{i} \mathrm{~d}}, d_{\mathrm{s}_{i} \mathrm{r}}$ and $d_{\mathrm{rd}}$ the distance from source $\mathrm{s}_{i}$ to the destination, from source $s_{i}$ to the relay, and from the relay to the destination, respectively. We also make the following assumptions:

A1 All sources are at the same distance from the destination, i.e., $d_{\mathrm{s}_{i} \mathrm{~d}}=d_{\mathrm{sd}} \forall i$. Therefore, $\gamma_{\mathrm{s}_{i} \mathrm{~d}}=\gamma_{\mathrm{sd}}$ for all sources $\mathrm{s}_{i}$.

A2 The relay is closer to the sources than to the destination. This is a favorable and necessary assumption for decodeand-forward techniques, since it guarantees a low error probability at the relay. Moreover, we assume that $d_{\mathrm{s}_{i} \mathrm{r}}=$ $d_{\mathrm{sr}}$ for all sources $\mathrm{s}_{i}$, i.e., $\gamma_{\mathrm{s}_{i} \mathrm{r}}=\gamma_{\mathrm{sr}} \forall i$.

A3 The SNR of the three channels under consideration are linked by

$$
\begin{gathered}
\gamma_{\mathrm{sr}}=g_{\mathrm{sr}} \gamma_{\mathrm{sd}}, \\
\gamma_{\mathrm{rd}}=g_{\mathrm{rd}} \gamma_{\mathrm{sd}},
\end{gathered}
$$

where the gains $g_{\mathrm{sr}}$ and $g_{\mathrm{rd}}$ are due to shorter transmission distances and are given by $g_{\mathrm{sr}}=\left(d_{\mathrm{sd}} / d_{\mathrm{sr}}\right)^{n}$ and $g_{\mathrm{rd}}=\left(d_{\mathrm{sd}} / d_{\mathrm{rd}}\right)^{n}$, respectively. $n$ denotes the path-loss exponent and it is often assumed to be $2 \leq n \leq 6[9$, 10]. Here, we consider a path-loss exponent of $n=3.52$ [11].

The received observations at the the relay and at the destination can be written as

$$
\begin{aligned}
\mathbf{y}_{\mathrm{s}_{i} \mathrm{r}} & =\sqrt{2 \gamma_{\mathrm{sr}}} \mathbf{H}_{\mathrm{s}_{i} \mathrm{r}} \check{\mathbf{x}}_{i}+\mathbf{n}_{\mathrm{s}_{i} \mathrm{r}}, \\
\mathbf{y}_{\mathrm{s}_{i} \mathrm{~d}} & =\sqrt{2 \gamma_{\mathrm{sd}}} \mathbf{H}_{\mathrm{s}_{i} \mathrm{~d}} \check{\mathbf{x}}_{i}+\mathbf{n}_{\mathrm{s}_{i} \mathrm{~d}}, \\
\mathbf{y}_{r d} & =\sqrt{2 \gamma_{\mathrm{rd}}} \mathbf{H}_{\mathrm{rd}} \check{\mathbf{x}}_{\mathrm{r}}+\mathbf{n}_{\mathrm{rd}},
\end{aligned}
$$

where $\check{\mathrm{x}}$ is the BPSK modulated sequence of the binary sequence $\mathbf{x}, \mathbf{n}_{\mathrm{s}_{i} \mathrm{r}}, \mathbf{n}_{\mathrm{s}_{i} \mathrm{~d}}$ and $\mathbf{n}_{\mathrm{rd}}$ are AWGN noise vectors with zero-mean, unit-variance i.i.d. elements, and $\mathbf{H}_{\mathrm{s}_{i} \mathrm{r}}, \mathbf{H}_{\mathrm{s}_{i} \mathrm{~d}}$ and $\mathbf{H}_{\mathrm{rd}}$ are diagonal matrices with Rayleigh fading channel coefficients in the main diagonal and zeros everywhere else. In this paper we consider fully-interleaved Rayleigh fading (fast fading).

\section{B. Non-orthogonal channels}

For delay sensitive applications (e.g. speech and video transmission) non-orthogonal schemes are more advantageous. 
Here, we consider that the transmission time is divided into two time slots. The $q$ sources transmit simultaneously over a single communication channel during the first time slot, realizing a multiple-access channel (MAC). The relay operates in a half-duplex mode; it receives a superposition of the signals from all sources during the first time slot and transmits its own parity during the second time slot.

Several transmitting approaches can be used to deal with multiple-access interference at the destination, such as codedivision multiple-access (CDMA) [12] and interleave-division multiple-access [13]. IDMA involves a spreading operation of the data (as in CDMA) and an interleaving operation; separation between users is obtained by the use of a different interleaver for each user. A key advantage of IDMA over CDMA is that, in general, the complexity of a CDMA multiuser detector or soft interference cancelation detector is polynomial with the number of users [14]. In contrast, IDMA lends itself to a simple chip-by-chip detection algorithm whose complexity grows only linearly with the number of users $[13$, 15]. In this paper, we consider the use of IDMA for multiuser detection at the relay and at the destination. For an overview of the IDMA technique the reader is referred to [13,15-17].

As before, each source $\mathrm{s}_{i}$ encodes information sequence $\mathbf{u}_{\mathrm{s}_{i}}$ of length $k_{i}$ by encoder $\mathcal{C}_{i}$ of rate $R_{i}$ into codeword $\mathbf{x}_{\mathrm{s}_{i}}$ of length $n_{i}=k_{i} / R_{i}$. The sequences $\mathbf{x}_{\mathrm{s}_{i}}$ are then spread (by using a repetition code) into sequences $\mathbf{c}_{\mathrm{s}_{i}}$ of length $n_{i}^{\mathrm{sp}}=m n_{i}$, where $m$ is the spreading factor. The code rate after spreading is denoted by $R_{i}^{\mathrm{sp}}=R_{i} / \mathrm{m}$. Finally, each sequence $\mathbf{c}_{\mathrm{s}_{i}}$ is scrambled by interleaver $\pi_{i}$ into the so-called chip sequence $\tilde{\mathbf{c}}_{\mathrm{s}_{i}}$.

All sources transmit simultaneously during the first time slot. The received signals at the relay and at the destination $\left(\mathbf{y}_{\mathrm{sr}}\right.$ and $\mathbf{y}_{\mathrm{sd}}$, respectively) are the superposition of the chip sequences $\check{\tilde{\mathbf{c}}}_{\mathrm{s}_{i}}$ (weighted by the channel coefficients) from the different sources,

$$
\begin{aligned}
\mathbf{y}_{\mathrm{sr}} & =\sum_{i=1}^{q} \sqrt{2 \gamma_{\mathrm{sr}}} \mathbf{H}_{\mathrm{s}_{i} \mathrm{r}} \check{\tilde{\mathbf{c}}}_{\mathrm{s}_{i}}+\mathbf{n}_{\mathrm{sr}}, \\
\mathbf{y}_{\mathrm{sd}} & =\sum_{i=1}^{q} \sqrt{2 \gamma_{\mathrm{sd}}} \mathbf{H}_{\mathrm{s}_{i} \mathrm{~d}} \check{\tilde{\mathbf{c}}}_{\mathrm{s}_{i}}+\mathbf{n}_{\mathrm{sd}},
\end{aligned}
$$

where $\check{\tilde{\mathbf{c}}}_{\mathrm{s}_{i}}$ is the BPSK modulated sequence of the binary sequence $\tilde{\mathbf{c}}_{\mathrm{s}_{i}}$.

After multiuser detection and decoding, the relay generates the parity sequence $\mathrm{x}_{\mathrm{r}}$ and cooperates with the sources by forwarding $\mathrm{x}_{\mathrm{r}}$ to the destination during the second time slot. The received observation at the destination is described as

$$
\mathbf{y}_{\mathrm{rd}}=\sqrt{2 \gamma_{\mathrm{rd}}} \mathbf{H}_{\mathrm{rd}} \check{\mathbf{x}}_{\mathrm{r}}+\mathbf{n}_{\mathrm{rd}} .
$$

Finally, the destination applies joint multiuser detection/decoding of the $q$ sources by exploiting $\mathbf{y}_{\mathrm{sd}}$ and $\mathbf{y}_{\mathrm{rd}}$.

\section{ENCODING STRATEGIES AT THE RELAY}

In the following sections we describe the proposed distributed SCC for the case of orthogonal channels. The case of non-orthogonal channels is addressed in Section VII.

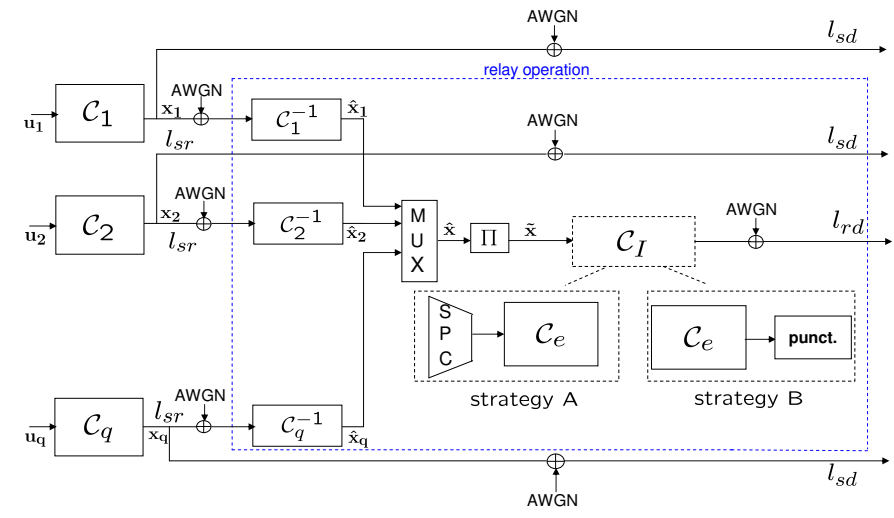

Fig. 2. Block diagram of the proposed distributed serially concatenated code. The relay processes information using strategy A or B.

The proposed relaying scheme can be regarded to as a decode-and-forward scheme. The relay receives a noisy observation of the codewords $\mathbf{x}_{\mathrm{s}_{i}}$ from the $q$ sources, it decodes them, and generates an estimate of the transmitted codewords. The estimated codewords $\hat{\mathbf{x}}_{i}$ are then properly combined, interleaved into $\tilde{\mathbf{x}}$ by an interleaver $\Pi$, and re-encoded by another encoder $\mathcal{C}_{\mathrm{e}}$ prior to transmission. We consider two encoding strategies at the relay.

\section{A. Strategy A}

The bits of codeword $\tilde{\mathbf{x}}$ at the output of the interleaver, of length $N=\sum_{i=1}^{q} n_{i}$ bits, are grouped into groups of $J$ bits and passed to a single parity check (SPC) circuit. This simply forms the sum modulo- 2 of the $J$ incoming bits, i.e., for each group of $J$ bits it generates a single bit as their modulo-2 sum. The codeword at the output of the SPC, of length $N_{\mathrm{SPC}}=N / J$ bits, is then encoded by a recursive convolutional encoder $\mathcal{C}_{\mathrm{e}}$ (typically of rate $R_{\mathrm{e}}=1$ ) and transmitted over the wireless channel. The effective code rate of the overall system is $R_{\text {eff }}=K / N^{\prime}$, where $K=\sum_{i=1}^{q} k_{i}$, $N^{\prime}=N+n_{\mathrm{r}}$ and $n_{\mathrm{r}}=N_{\mathrm{SPC}} / R_{\mathrm{e}}=N / R_{\mathrm{I}}$ is the length of the codeword transmitted by the relay, where $R_{\mathrm{I}}=J R_{\mathrm{e}}$. The proposed distributed coding scheme is depicted in Fig. 2.

\section{B. Strategy B}

The bits of the codeword $\tilde{\mathbf{x}}$ are encoded by a recursive inner encoder $\mathcal{C}_{\mathrm{e}}$ (typically of rate $R_{\mathrm{e}}=1$ ) heavily punctured to rate $R_{\mathrm{I}}=R_{\mathrm{e}} / \rho_{p}>1$ through a puncturer $\mathcal{P}$ and transmitted to the destination. A puncturer $\mathcal{P}$ is defined by a puncturing pattern $\mathbf{p}$ of puncturing period $N_{p}$. Let $\delta_{p}\left(0 \leq \delta_{p} \leq N_{p}\right)$ denote the number of bits remaining at the output of the puncturer within a puncturing period $N_{p}$. We denote by $\rho_{p}=\delta_{p} / N_{p}$ $\left(0 \leq \rho_{p} \leq 1\right)$ the permeability ratio of the puncturer, giving the ratio of bits that survive puncturing. Note that $\rho_{p}=0$ corresponds to the non-cooperation case. The effective code rate of the overall system is $R_{\text {eff }}=K / N^{\prime}$, where $N^{\prime}=N+n_{\mathrm{r}}$ and $n_{\mathrm{r}}=N / R_{\mathrm{I}}$. Note that while the encoder at the relay is of rate higher than one (and therefore non-invertible), the overall distributed coding scheme is of rate $R_{\text {eff }}<1$, which allows correct recovering of users data. The distributed coding scheme is depicted in Fig. 2. 
Parameters $J$ in strategy A and $\rho_{p}$ in strategy B determine the amount of redundancy transmitted by the relay, i.e., given $R_{i}$ and $q$ they determine the effective code rate of the overall system. $J$ and $\rho_{p}$ can be adjusted according to requirements in terms of performance, overall system rate and/or power constraints. Notice that strategy B allows for a finer control of the redundancy transmitted by the relay, if $N_{p}$ is large enough.

\section{Code optimization}

The performance of the proposed distributed codes depends on encoders $\mathcal{C}_{i}$ and $\mathcal{C}_{\mathrm{e}}$ and on $J$ and $\left(\mathbf{p}, \delta_{p}\right)$ for strategy $\mathrm{A}$ and strategy B, respectively. Given $q$ and the overall system rate $R_{\text {eff }}$, the goal is to optimize these parameters to ensure good performance in both the error floor and waterfall regions. For simplicity and flexibility, we consider rate- $1 / 2$ convolutional encoders for $\mathcal{C}_{i}$, which can be punctured to the desired rate $R_{i}$.

A joint optimization of the encoders polynomials and of $J$ and $\left(\mathbf{p}, \delta_{p}\right)$ to optimize the error floor and the convergence threshold is prohibitively complex. Therefore, we consider the following strategy, yet feasible: first, the encoder polynomials of $\mathcal{C}_{i}$ and $\mathcal{C}_{\mathrm{e}}$ (and the puncturing pattern $\mathbf{p}$ for strategy $\mathrm{B}$ ) are chosen according to design criteria for the error floor [18]. Then, time allocation, i.e., parameters $J$ and $\rho_{p}$ and the rates $R_{i}$ are optimized according to other criteria. In this paper, we optimize the time allocation according to two criteria: in Section V-B we analytically optimize the time allocation to maximize the achievable rates; in Section VI we use EXIT charts to minimize the decoding thresholds.

For the examples in this paper, we consider the same encoder for all sources. For strategy A, the same optimal (in terms of error floor) 4-state, rate-1/2, feedforward convolutional encoder with generator polynomials $(5,7)_{8}$ in octal notation is used at each source, and the 4-state, rate-1, recursive convolutional encoder with generator polynomial $(3 / 7)_{8}$ is used for $\mathcal{C}_{\mathrm{e}}$ [19]. For strategy $\mathrm{B}$, the same optimal 4state, rate-1/2, recursive convolutional encoder with generator polynomials $(1,5 / 7)_{8}{ }^{1}$ is used at each source [20]. The optimal 4-state, rate-1, recursive convolutional encoder with generator polynomial $(5 / 7)_{8}$ is used at the relay [20]. Also, the puncturing pattern $\mathbf{p}$ in [20], which is optimized for the error floor, is used. Notice that for both strategies, encoder $\mathcal{C}_{\mathrm{e}}$ must be recursive to provide an interleaver gain. Notice also that for strategy A we do not use the optimal encoder for $\mathcal{C}_{\mathrm{e}}$, but instead we use the encoder $(3 / 7)_{8}$ proposed in [19]. This is because the optimal encoder entails some loss in terms of convergence.

\section{THE RELAY NETWORK AS A SERIALLY CONCATENATED} CODE

The proposed distributed coding scheme can be seen as a SCC where the outer encoder accounts for the encoders $\mathcal{C}_{i}$ of the $q$ sources, and the inner encoder is the relay. The equivalent

\footnotetext{
${ }^{1}$ According to [18] it can be convenient to choose as outer code a nonrecursive encoder. Here, for simplicity, we considered identical encoders for $\mathcal{C}_{i}$ and $\mathcal{C}_{\mathrm{e}}$. Moreover, it is worth mentioning that the difference in performance is marginal.
}

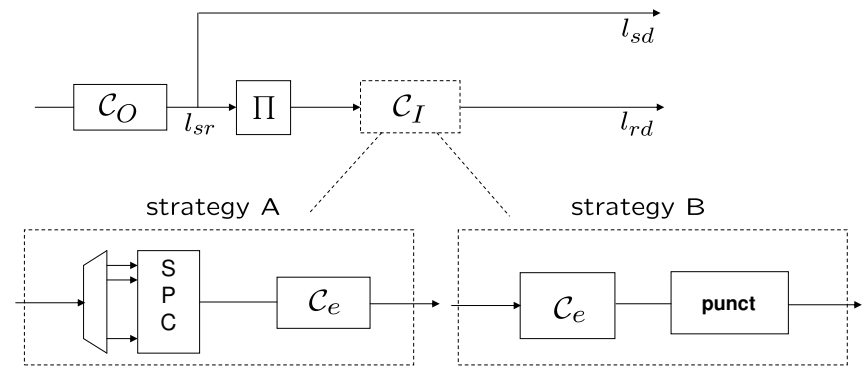

Fig. 3. Equivalent representation of the wireless relay network of Fig. 2.

representation is depicted in Fig. 3. The information sequences $\mathbf{u}_{\mathrm{s}_{i}}$ are concatenated and encoded by the outer encoder $\mathcal{C}_{\mathrm{O}}$. Note that $\mathcal{C}_{\mathrm{O}}$ is time-variant if encoders $\mathcal{C}_{i}$ are different. The encoded information is transmitted over a wireless channel with SNR $\gamma_{\mathrm{sd}}$, corresponding to the direct link between the sources and the destination, $l_{\mathrm{sd}}$. It is also interleaved and reencoded by the inner encoder $\mathcal{C}_{\mathrm{I}}$, of rate $R_{\mathrm{I}}$, which implements strategy A or B. The resulting codeword is transmitted over a wireless channel with SNR $\gamma_{\mathrm{rd}}$. Note that the codeword at the input of the inner encoder may contain errors.

From the equivalent representation in Fig. 3 the SCC resulting from strategy $\mathrm{A}$ is similar to the coding scheme introduced in [19], nicknamed Flexicode. In [19] a SPC circuit is used before the inner encoder of a SCC to increase the code rate while preserving very good performance in the error floor. Moreover, a copy of the input data is sent directly to the channel, i.e., the code is systematic. Here, the key idea is to use a SPC to group several users and to control the rate of the relay-to-destination link, instead of increasing the code rate. The overall scheme is therefore a form of (non-systematic) distributed Flexicode. On the other hand, the SCC resulting from strategy B is similar to the SCC scheme proposed in [20]. In [20] a SCC was proposed where the inner encoder was heavily punctured beyond the unitary rate. The proposed scheme allows keeping a very low error floor for a wide range of code rates since the interleaver gain is preserved, and achieves much better performance than standard SCCs, especially for high rates. Here, the level of puncturing of the inner code is used to group several users instead of increasing the code rate.

The coding schemes in [19] and [20] are the best known SCCs for high rates. They also allow a high flexibility in terms of code rate. In terms of the wireless network of Fig. 1 high rates correspond to a high number of users. Therefore, the proposed schemes are expected to perform very well even for a large number of users.

\section{A. Decoding of the distributed serially concatenated code}

According to the equivalent representation in Fig. 3, decoding of users data can be performed using a decoding strategy that resembles the decoding of a SCC: decoding of the information of all sources can be done jointly, exploiting the redundancy provided by the relay in an iterative fashion. One possible (but not unique) scheduling for strategies A and $\mathrm{B}$ is as follows: 
- The relay-to-destination channel metrics are fed to the inner decoder, and the source-to-destination channel metrics are fed to each source decoder.

- The receiver decodes first the sources and generates extrinsic information on codeword $\hat{\mathbf{x}}$.

- The inner encoder (the concatenation of the SPC and encoder $\mathcal{C}_{\mathrm{e}}$ and the concatenation of encoder $\mathcal{C}_{\mathrm{e}}$ and the puncturer for strategy $\mathrm{A}$ and $\mathrm{B}$, respectively) is then decoded by using the extrinsic information on $\hat{\mathbf{x}}$ (properly interleaved) provided by the decoders of the sources as a priori information, and generates extrinsic information on $\tilde{\mathbf{x}}$ which will be used as a priori information (after deinterleaving) by the decoders of the sources at the next iteration.

- The process is repeated until the maximum number of iterations is reached or an early stopping criterion is fulfilled.

\section{B. The overall network performing as a parallel concatenated code}

Instead of generating an estimate of codewords $\mathbf{x}_{i}$ the relay may estimate information words $\mathbf{u}_{i}$. The estimates $\hat{\mathbf{u}}_{i}$ are then properly combined, interleaved and encoded by encoder $\mathcal{C}_{\mathrm{e}}$ using strategy $\mathrm{A}$ or $\mathrm{B}$ as described before. The resulting relay network effectively realizes a (asymmetric) distributed parallel concatenated code (PCC); the upper encoder of the PCC accounts for encoders $\mathcal{C}_{i}$ of the $q$ sources and the lower encoder corresponds to the relay.

\section{INFORMATION-THEORETIC LIMITS}

In this section we compute the achievable rates for the multi-source relay network of Fig. 1, following the approach proposed in [21]. In [21] the authors considered the achievable decode-and-forward rate of a 2-user system assuming the time division multiple access relay channel (MARC) model with optimized allocation of the transmission time. In the following, we consider for simplicity the time division MARC with two sources and one relay. We will then give results for the generalized multi-user case.

Let $k_{i}, n_{i}, i=1,2, n_{\mathrm{r}}$ and $N^{\prime}$ be as defined in Sections II and III. We define the rate for source $s_{i}$ as $R_{i}^{\prime}=k_{i} / N^{\prime}$. With these definitions it follows $K=k_{1}+k_{2}, N^{\prime}=n_{1}+n_{2}+n_{\mathrm{r}}$ and the rate of the overall system is $R_{\text {eff }}=R_{1}^{\prime}+R_{2}^{\prime}=K / N^{\prime}$. The information data of sources $s_{1}$ and $s_{2}$ can be decoded reliably at the destination if the following inequalities hold [21]:

$$
\begin{aligned}
k_{1} & \leq n_{1} C\left(\gamma_{\mathrm{s}_{1} \mathrm{r}}\right) \\
k_{2} & \leq n_{2} C\left(\gamma_{\mathrm{s}_{2} \mathrm{r}}\right) \\
k_{1} & \leq n_{1} C\left(\gamma_{\mathrm{s}_{1} \mathrm{~d}}\right)+n_{\mathrm{r}} C\left(\gamma_{\mathrm{rd}}\right) \\
k_{2} & \leq n_{2} C\left(\gamma_{\mathrm{s}_{2} \mathrm{~d}}\right)+n_{\mathrm{r}} C\left(\gamma_{\mathrm{rd}}\right) \\
k_{1}+k_{2} & \leq n_{1} C\left(\gamma_{\mathrm{s}_{1} \mathrm{~d}}\right)+n_{2} C\left(\gamma_{\mathrm{s}_{2} \mathrm{~d}}\right)+n_{\mathrm{r}} C\left(\gamma_{\mathrm{rd}}\right),
\end{aligned}
$$

where $C$ denotes the instantaneous capacity.

We define the time allocated to $\mathrm{s}_{1}, \mathrm{~s}_{2}$ and to the relay as $\theta_{1}=n_{1} / N^{\prime}, \theta_{2}=n_{2} / N^{\prime}$ and $\theta_{\mathrm{r}}=n_{\mathrm{r}} / N^{\prime}$, respectively.
Notice that $\theta_{1}+\theta_{2}+\theta_{\mathrm{r}}=1$. From (5) the achievable rate $R_{1}^{\prime}$ is given by

$$
\begin{aligned}
& R_{1}^{\prime}=\min \left\{\theta_{1} C\left(\gamma_{\mathrm{s}_{1} \mathrm{r}}\right), \theta_{2} C\left(\gamma_{\mathrm{s}_{2} \mathrm{r}}\right) / \sigma,\right. \\
& \quad \theta_{1} C\left(\gamma_{\mathrm{s}_{1} \mathrm{~d}}\right)+\left(1-\theta_{1}-\theta_{2}\right) C\left(\gamma_{\mathrm{rd}}\right), \\
& \quad\left(\theta_{2} C\left(\gamma_{\mathrm{s}_{2} \mathrm{~d}}\right)+\left(1-\theta_{1}-\theta_{2}\right) C\left(\gamma_{\mathrm{rd}}\right)\right) / \sigma, \\
& \left.\quad\left(\theta_{1} C\left(\gamma_{\mathrm{s}_{1} \mathrm{~d}}\right)+\theta_{2} C\left(\gamma_{\mathrm{s}_{2} \mathrm{~d}}\right)+\left(1-\theta_{1}-\theta_{2}\right) C\left(\gamma_{\mathrm{rd}}\right)\right) /(1+\sigma)\right\},
\end{aligned}
$$

where $\sigma=R_{2}^{\prime} / R_{1}^{\prime}$. Here, we consider a symmetric MARC, i.e., $\sigma=1$.

\section{A. Equal time allocation}

Consider equal time allocation for the two sources and the relay $\left(\theta_{1}=\theta_{2}=\theta_{\mathrm{r}}=1 / 3\right)$, which corresponds to the case where the relay and the sources transmit the same amount of information. Also, assume $\gamma_{\mathrm{s}_{1} \mathrm{r}}=\gamma_{\mathrm{s}_{2} \mathrm{r}}$ and $\gamma_{\mathrm{s}_{1} \mathrm{~d}}=\gamma_{\mathrm{s}_{2} \mathrm{~d}}$ (Assumption A2). The achievable rates $R_{1}^{\prime}$ and $R_{2}^{\prime}$ are given by

$$
R_{1}^{\prime}=R_{2}^{\prime}=\min \left\{\frac{1}{3} C\left(\gamma_{\mathrm{sr}}\right), \frac{1}{3} C\left(\gamma_{\mathrm{sd}}\right)+\frac{1}{6} C\left(\gamma_{\mathrm{rd}}\right)\right\} .
$$

A similar analysis can be performed for the multi-source scenario with $q$ sources by generalizing (5) and (6). The achievable rates for sources $\mathrm{s}_{i}, i=1, \ldots, q$, with equal time allocation $\left(\theta_{i}=\theta_{\mathrm{r}}=1 /(q+1)\right)$ and under the assumption that all sources transmit at the same rate $\left(R_{i}^{\prime}=R_{\mathrm{s}}^{\prime} \quad \forall i\right)$ are given by

$$
\begin{aligned}
R_{i}^{\prime} & =R_{\mathrm{s}}^{\prime} \\
& =\min \left\{\frac{1}{q+1} C\left(\gamma_{\mathrm{sr}}\right), \frac{1}{q+1} C\left(\gamma_{\mathrm{sd}}\right)+\frac{1}{q(q+1)} C\left(\gamma_{\mathrm{rd}}\right)\right\} .
\end{aligned}
$$

The rate of the overall system, also referred to as the sum rate, is $R_{\text {eff }}=q R_{s}^{\prime}$.

In Table I we report the minimum value of $\gamma_{\mathrm{sd}}^{\mathrm{b}}$, denoted by $\gamma_{\text {min,eq }}^{\mathrm{b}}$, such that a system rate $R_{\text {eff }}=\frac{q}{2(q+1)}$ is achieved for a Rayleigh fast fading channel and several values of $q$ (column 4). Here, we assume that each source transmits with a code rate $R=1 / 2$. Therefore, with equal time allocation, $R_{\text {eff }}=\frac{q k}{q 2 k+2 k}=\frac{q}{2(q+1)} \cdot \gamma_{\mathrm{sd}}^{\mathrm{b}}=\gamma_{\mathrm{sd}} / R_{\mathrm{eff}}$ denotes the SNR (in $\mathrm{dB}$ ) per information bit. In the computation, we assumed $d_{\mathrm{sr}}=(1 / 4) d_{\mathrm{sd}}$ and $d_{\mathrm{rd}}=(3 / 4) d_{\mathrm{sd}}$.

\section{B. Optimal time allocation}

The transmission time allocated to the sources and to the relay can be optimized to maximize the achievable rates. For a 2-user relay network, the optimal time allocation can be derived so that $R_{1}^{\prime}$ is maximized, subject to some ratio $\sigma=$ $R_{1}^{\prime} / R_{2}^{\prime}$ between the achievable rates $R_{1}^{\prime}$ and $R_{2}^{\prime}$ for user one and two, respectively, is satisfied [21]:

$$
\begin{aligned}
& {\left[\theta_{1}^{\text {opt }}, \theta_{2}^{\text {opt }}\right]=\arg \max _{\left[\theta_{1}, \theta_{2}\right]} R_{1}^{\prime}} \\
& \text { subject to } \sigma=R_{2}^{\prime} / R_{1}^{\prime} \text { and } \theta_{\mathrm{r}}=1-\theta_{1}-\theta_{2} .
\end{aligned}
$$


TABLE I

CONVERGENCE THRESHOLDS AND MINIMUM $\gamma_{\mathrm{sd}}^{\mathrm{b}}$ THAT ACHIEVES A RATE OF $R_{\mathrm{eff}}=\frac{q}{2(q+1)}$ FOR THE DISTRIBUTED SERIALLY CONCATENATED SCHEMES FOR DIFFERENT NUMBER OF USERS

\begin{tabular}{ccccccccccc}
\hline & Threshold (A) & Threshold (B) & $\gamma_{\text {min }, \text { eq }}^{\mathrm{b}}$ & Threshold ${ }^{*}(\mathrm{~B})$ & $\gamma_{\text {min }, \text { opt }}^{\mathrm{b}}$ & $\theta_{s}^{\text {opt }}$ & $\theta_{\mathrm{r}}^{\text {opt }}$ & $\theta_{\mathrm{s}}^{*}$ & $\theta_{\mathrm{r}}^{*}$ & $R_{s}^{*}$ \\
\hline \hline$q=2$ & $0.47 \mathrm{~dB}$ & $0.77 \mathrm{~dB}$ & $-1.0982 \mathrm{~dB}$ & $-0.75 \mathrm{~dB}$ & $-2.562262 \mathrm{~dB}$ & 0.172112 & 0.655776 & 0.2564 & 0.4871 & 0.65 \\
$q=4$ & $1.37 \mathrm{~dB}$ & $1.57 \mathrm{~dB}$ & $0.0658 \mathrm{~dB}$ & $-0.35 \mathrm{~dB}$ & $-1.723154 \mathrm{~dB}$ & 0.102246 & 0.591016 & 0.1250 & 0.5000 & 0.8 \\
$q=8$ & $2.32 \mathrm{~dB}$ & $2.42 \mathrm{~dB}$ & $0.8604 \mathrm{~dB}$ & $0.3 \mathrm{~dB}$ & $-1.129677 \mathrm{~dB}$ & 0.056535 & 0.54772 & 0.0694 & 0.4444 & 0.8 \\
$q=20$ & $3.52 \mathrm{~dB}$ & $3.52 \mathrm{~dB}$ & $1.4152 \mathrm{~dB}$ & $0.75 \mathrm{~dB}$ & $-0.692474 \mathrm{~dB}$ & 0.024164 & 0.48328 & 0.0297 & 0.4047 & 0.8 \\
$q=30$ & $4.00 \mathrm{~dB}$ & $4.00 \mathrm{~dB}$ & $1.5406 \mathrm{~dB}$ & $0.9 \mathrm{~dB}$ & $-0.58547 \mathrm{~dB}$ & 0.016360 & 0.5092 & 0.0202 & 0.3952 & 0.8 \\
$q=50$ & $4.66 \mathrm{~dB}$ & $4.66 \mathrm{~dB}$ & $1.6618 \mathrm{~dB}$ & $1.0 \mathrm{~dB}$ & $-0.483338 \mathrm{~dB}$ & 0.009939 & 0.50305 & 0.0122 & 0.3872 & 0.8 \\
$q=100$ & $5.25 \mathrm{~dB}$ & $5.40 \mathrm{~dB}$ & $1.7443 \mathrm{~dB}$ & $1.05 \mathrm{~dB}$ & $-0.412715 \mathrm{~dB}$ & 0.005017 & 0.4983 & 0.0062 & 0.3812 & 0.8 \\
\hline
\end{tabular}

If conditions $C\left(\gamma_{\mathrm{s}_{1} \mathrm{~d}}\right)<C\left(\gamma_{\mathrm{rd}}\right), C\left(\gamma_{\mathrm{s}_{2} \mathrm{~d}}\right)<C\left(\gamma_{\mathrm{rd}}\right)$, $C\left(\gamma_{\mathrm{s}_{1} \mathrm{~d}}\right)<C\left(\gamma_{\mathrm{s}_{1} \mathrm{r}}\right)$ and $C\left(\gamma_{\mathrm{s}_{2} \mathrm{~d}}\right)<C\left(\gamma_{\mathrm{s}_{2} \mathrm{r}}\right)$ hold, a closedform expression for the optimal time allocation can be derived [21]. The optimal time allocation parameters are given by

$$
\theta_{1}^{\mathrm{opt}}=\frac{C\left(\gamma_{\mathrm{rd}}\right)}{(1+\sigma \alpha) C\left(\gamma_{\mathrm{rd}}\right)+(1+\sigma) C\left(\gamma_{\mathrm{s}_{1} \mathrm{r}}\right)-C\left(\gamma_{\mathrm{s}_{1} \mathrm{~d}}\right)-\sigma \alpha C\left(\gamma_{\mathrm{s}_{2} \mathrm{~d}}\right)}
$$

and

$$
\theta_{2}^{\mathrm{opt}}=\theta_{1}^{\mathrm{opt}} \sigma \alpha,
$$

where $\alpha=C\left(\gamma_{\mathrm{s}_{1} \mathrm{r}}\right) / C\left(\gamma_{\mathrm{s}_{2} \mathrm{r}}\right)$.

The achievable rates $R_{1}^{\prime}$ and $R_{2}^{\prime}$ are given by

$$
R_{1}^{\prime}=\frac{C\left(\gamma_{\mathrm{s}_{1} \mathrm{r}}\right) C\left(\gamma_{\mathrm{rd}}\right)}{(1+\sigma \alpha) C\left(\gamma_{\mathrm{rd}}\right)+(1+\sigma) C\left(\gamma_{\mathrm{s}_{1} \mathrm{r}}\right)-C\left(\gamma_{\mathrm{s}_{1} \mathrm{~d}}\right)-\sigma \alpha C\left(\gamma_{\mathrm{s}_{2} \mathrm{~d}}\right)}
$$

and

$$
R_{2}^{\prime}=\sigma R_{1}^{\prime}
$$

In this paper, we consider a symmetric MARC $(\sigma=1)$, and $\gamma_{\mathrm{s}_{1} \mathrm{r}}=\gamma_{\mathrm{s}_{2} \mathrm{r}}$ and $\gamma_{\mathrm{s}_{1} \mathrm{~d}}=\gamma_{\mathrm{s}_{2} \mathrm{~d}}$ (Assumption A2), from which it follows $\alpha=1$. We obtain

$$
\theta_{1}^{\mathrm{opt}}=\theta_{2}^{\mathrm{opt}}=\frac{1}{2} \frac{C\left(\gamma_{\mathrm{rd}}\right)}{C\left(\gamma_{\mathrm{rd}}\right)+C\left(\gamma_{\mathrm{sr}}\right)-C\left(\gamma_{\mathrm{sd}}\right)}
$$

and

$$
R_{1}^{\prime}=R_{2}^{\prime}=\frac{1}{2} \frac{C\left(\gamma_{\mathrm{sr}}\right) C\left(\gamma_{\mathrm{rd}}\right)}{C\left(\gamma_{\mathrm{rd}}\right)+C\left(\gamma_{\mathrm{sr}}\right)-C\left(\gamma_{\mathrm{sd}}\right)} .
$$

The optimal time allocation and the achievable rates for the symmetric MARC with $q$ sources can be derived in a similar way. The optimal time allocation $\theta_{i}$ for source $\mathrm{s}_{i}$ and relay $\mathrm{r}$ is given by

$$
\theta_{i}^{\mathrm{opt}}=\frac{1}{q} \frac{C\left(\gamma_{\mathrm{rd}}\right)}{C\left(\gamma_{\mathrm{rd}}\right)+C\left(\gamma_{\mathrm{sr}}\right)-C\left(\gamma_{\mathrm{sd}}\right)}=\theta_{\mathrm{s}}^{\mathrm{opt}} \forall i
$$

and

$$
\theta_{\mathrm{r}}^{\mathrm{opt}}=1-\sum_{i=1}^{q} \theta_{i}^{\mathrm{opt}}=1-q \theta_{\mathrm{s}}^{\mathrm{opt}},
$$

while the achievable rate for source $\mathrm{s}_{i}$ is given by

$$
R_{i}^{\prime}=R_{s}^{\prime}=\frac{1}{q} \frac{C\left(\gamma_{\mathrm{sr}}\right) C\left(\gamma_{\mathrm{rd}}\right)}{C\left(\gamma_{\mathrm{rd}}\right)+C\left(\gamma_{\mathrm{sr}}\right)-C\left(\gamma_{\mathrm{sd}}\right)} \quad \forall i .
$$

In the Appendix, we have summarized the optimization of the time allocation for the symmetric MARC and $q=2$.

From (18), we observe that the MARC achieves the same sum capacity of the relay channel and therefore the optimization problem can be treated as a capacity optimization problem for a single user relay channel [21].

In Table I we report the optimal time allocation parameters $\theta_{\mathrm{s}}^{\text {opt }}, \theta_{\mathrm{r}}^{\text {opt }}$ and the minimum value of $\gamma_{\mathrm{sd}}^{\mathrm{b}}$, denoted by $\gamma_{\mathrm{min}, \mathrm{opt}}^{\mathrm{b}}$,

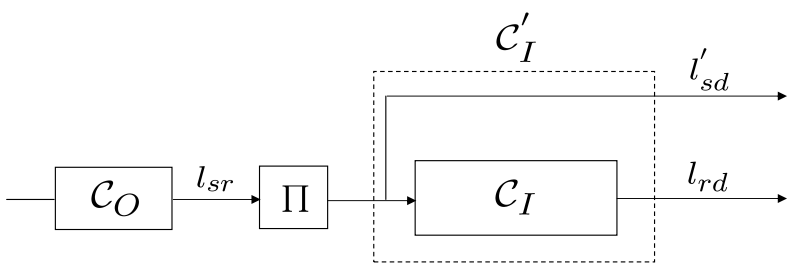

Fig. 4. Another equivalent representation of the distributed SCC of Fig. 2.

such that a system rate $R_{\text {eff }}=\frac{q}{2(q+1)}$ is achieved over a fast fading channel and several values of $q$ (column 6 ). In the computation, we assumed $d_{\mathrm{sr}}=(1 / 4) d_{\mathrm{sd}}$ and $d_{\mathrm{rd}}=(3 / 4) d_{\mathrm{sd}}$. An improvement of $1.4-2.2 \mathrm{~dB}$ with respect to equal time allocation is observed, depending on the number of sources.

For optimal time allocation the rate $R_{\mathrm{I}}$ of the relay encoder of the proposed distributed SCCs is given by $R_{\mathrm{I}}=1 /(1-$ $\left.q \theta_{\mathrm{s}}^{\mathrm{opt}}\right)-1$. Also, for a given overall system rate $R_{\mathrm{eff}}=\frac{q}{2(q+1)}$ the rate of each encoder $\mathcal{C}_{i}$ must satisfy $R_{i}=R_{\mathrm{s}}=\frac{1}{2 \theta_{\mathrm{s}}^{\text {opt }}(q+1)}$ (assuming all users transmitting at the same rate). Varying $R_{\mathrm{I}}$ is very simple by tuning parameters $J$ and $\rho_{p}$ in strategy A and strategy B, respectively (see Section III), while different code rates $R_{i}$ can be obtained by puncturing a mother encoder $\mathcal{C}_{i}$. Therefore, the proposed scheme offers a high flexibility. Notice that strategy B allows for a finer approximation of $R_{\mathrm{I}}$, since $\rho_{p}$ provides a high resolution if $N_{p}$ is large enough.

From the values of $\theta_{\mathrm{s}}^{\text {opt }}$ in Table I, we obtain that the optimal rate $R_{\mathrm{S}}$ for the sources is $\sim 0.98$ for all values of q. Unfortunately, it is hard to design good codes for such a high rate. In particular, a heavy puncturing of a convolutional code leads to a poor code and thereby to poor performance in the error floor. In the next section, we use EXIT charts to optimize the time allocation.

\section{EXIT CHARTS ANALYSIS}

The equivalent representation of the wireless relay network in Fig. 3 allows applying standard tools for the analysis of SCCs to analyze the performance of the proposed distributed coding scheme. In this section, we compute the convergence thresholds of the proposed distributed SCCs through an extrinsic information transfer (EXIT) chart analysis [22]. For convenience, the equivalent representation of the multi-source wireless network in Fig. 3 is further redrawn in the form of Fig. 4, where the outer encoder is not directly connected to the channel. Then, the contribution of the direct link between the sources and the destination is moved to the inner encoder $\mathcal{C}_{\mathrm{I}}^{\prime}$ (through its systematic branch). Note that the link $l_{\mathrm{sd}}$ corresponds to the concatenation of $l_{\mathrm{sr}}$ and $l_{\mathrm{sd}}^{\prime}$ in Fig. 4. Also, 


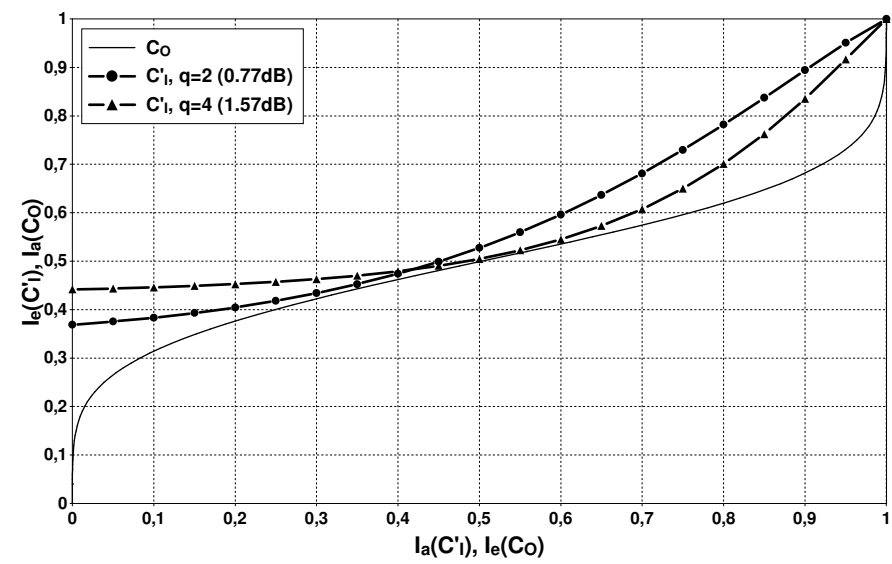

Fig. 5. EXIT chart of the proposed distributed turbo-like code, strategy B, for $q=2$ and $q=4$ users. Fast fading Rayleigh channel. $R_{\mathrm{eff}}=\frac{q}{2(q+1)}$ The convergence threshold for $q=2$ is $\gamma_{\mathrm{sd}}^{\mathrm{b}}=0.77 \mathrm{~dB}$ and for $q=4$ is $\gamma_{\mathrm{sd}}^{\mathrm{b}}=1.57 \mathrm{~dB}$.

note that for analysis purposes, representations in Figs. 3 and 4 are equivalent.

We assume that $\gamma_{\mathrm{sr}}$ is high enough, so that no errors occur at the relay. In this case, the convergence behavior of the distributed turbo-like code in Fig. 2 can be tracked using standard EXIT charts by plotting in a single chart the EXIT curve of the outer encoder $\mathcal{C}_{O}$ (which is now independent of $\gamma_{\mathrm{sd}}$ ) and the EXIT curve of the inner encoder $\mathcal{C}_{\mathrm{I}}^{\prime}$ which depends on both $\gamma_{\mathrm{sd}}$ and $\gamma_{\mathrm{rd}}$. In Fig. 5 we plot the EXIT charts of the proposed distributed coding scheme for strategy B and $q=2$ and $q=4$ users over a fast fading Rayleigh channel. Consider first that the relay and the sources transmit the same amount of symbols (equal time allocation), and $R_{i}=R_{\mathrm{s}}=1 / 2$. This is equivalent to set $\rho_{p}=1 / q$, i.e., only one bit out of $q$ at the output of encoder $\mathcal{C}_{\mathrm{e}}$ is preserved. Note that with this choice of $\rho_{p}$ the rate of the link $l_{\text {rd }}$ is kept constant independently of the number of users, and $R_{\mathrm{eff}}=\frac{q}{2(q+1)}$. Finally, we assume that $d_{\mathrm{sr}}=(1 / 4) d_{\mathrm{sd}}$ and $d_{\mathrm{rd}}=(3 / 4) d_{\mathrm{sd}}$, which translate into $g_{\mathrm{sr}}=131.6(21.19 \mathrm{~dB})$ and $g_{\mathrm{rd}}=2.75(4.4 \mathrm{~dB})$, respectively (see Section II). A tunnel between the EXIT curve of the outer encoder and the EXIT curve of the inner encoder opens at $\gamma_{\mathrm{sd}}^{\mathrm{b}}=0.77 \mathrm{~dB}$ and $\gamma_{\mathrm{sd}}^{\mathrm{b}}=1.57 \mathrm{~dB}$ for $q=2$ and $q=4$ users, respectively, indicating convergence around these values. The estimated convergence thresholds are given in Table I for $q=2,4,8,20,30,50$ and 100 users and strategies $\mathrm{A}$ and B. Also, for equal time allocation, $J=q$. The estimated convergence thresholds are similar for both strategies. We observed that the predicted thresholds match with simulation results for very long block sizes even if (some) errors occur at the relay (i.e., when $\gamma_{\mathrm{sr}}$ is limited). The proposed distributed SCCs perform within $1.5-2.0 \mathrm{~dB}$ from capacity for a number of users up to $q=20$. For higher $q$ the gap to capacity increases.

We used EXIT charts to optimize the time allocation for strategy B, which allows for a finer approximation of the optimal time allocation. In particular, we assume that all sources transmit at the same rate $R_{i}=R_{\mathrm{s}}$ and we find the pair $\left(R_{\mathrm{s}}, \rho_{p}\right)$ (equivalently $\left(R_{\mathrm{s}}, R_{I}\right)$ ) which minimizes the convergence threshold. To simplify the search, we limit $R_{\mathrm{S}}$ to the set $\{0.5,0.55, \ldots, 0.95\}$. The rate $R_{\mathrm{S}}$ is obtained by randomly puncturing the rate- $1 / 2$ convolutional encoder. We denote by $R_{\mathrm{s}}^{*}$ the value of $R_{\mathrm{S}}$ which minimizes the convergence threshold and by $\theta_{\mathrm{s}}^{*}$ and $\theta_{\mathrm{r}}^{*}$ the corresponding time allocation between the sources and the relay (notice that given $R_{\mathrm{s}}^{*}$ and $R_{\text {eff }}, \rho_{p}$ is fixed). In Table I we report the convergence thresholds for the case that the transmission time is allocated according to $\theta_{\mathrm{s}}^{*}$ and $\theta_{\mathrm{r}}^{*}$. We also report the value of $R_{\mathrm{s}}^{*}$. The same overall system rate $R_{\mathrm{eff}}=\frac{q}{2(q+1)}$ is assumed for all values of $q$. A significant gain with respect to equal time allocation is observed. Notice that the gain significantly increases with increasing value of $q$. Also, the gap to capacity is within $1.4-1.9 \mathrm{~dB}$, depending on the number of sources.

\section{Multi-SOURCE SCENARIO WITH MULTIPLE-ACCESS INTERFERENCE}

The proposed distributed SCCs for the case of nonorthogonal channels are identical to those of the scenario with orthogonal channels. However, in this case the effective overall system rate is different due to the spreading process and the fact that all sources transmit simultaneously. We recall that the relay estimates codewords $\mathbf{x}_{i}$ of the $q$ sources. The estimates $\hat{\mathbf{x}}_{i}$ are then properly combined, interleaved into $\tilde{\mathbf{x}}$ and encoded using strategy A or strategy B. Here, codeword $\tilde{\mathbf{x}}$ at the output of interleaver $\Pi$ has length $N=\sum_{i=1}^{q} n_{i}$. Therefore we have $n_{\mathrm{r}}=N / R_{\mathrm{I}}$ and the effective overall system rate is $R_{\text {eff }}=K / N^{\prime}$ where now $N^{\prime}=n_{i}^{\mathrm{sp}}+n_{\mathrm{r}}$.

Since all sources transmit simultaneously, a multiuser detector at the relay and at the destination are required to distinguish between signals from different users. We assume perfect synchronization. The relay uses an IDMA receiver that implements the chip-by-chip multiuser detection algorithm described in [16] to estimate codewords $\mathbf{x}_{i}$. The IDMA receiver consists of an elementary signal estimator (ESE) and $q a$ posteriori probability (APP) decoders $\mathcal{C}_{i}^{-1}$, corresponding to encoders $\mathcal{C}_{i}$. The ESE and decoders $\mathcal{C}_{i}^{-1}$ exchange soft information on codewords $\mathbf{x}_{i}$ in an iterative fashion. A decoding iteration consists of a single activation of the ESE and of the $q$ decoders $\mathcal{C}_{i}^{-1}, i=1, \ldots, q$, in this order. This process continues iteratively until the maximum number of iterations is reached or an early stopping rule criterion is fulfilled.

On the other hand, the destination receives the superposition of the signals from the $q$ sources, $\mathbf{y}_{\mathrm{sd}}$, and the noisy observation of the extra parity forwarded by the relay, $\mathbf{y}_{\mathrm{rd}}$. It then estimates information words $\mathbf{u}_{i}$ by exploiting jointly $\mathbf{y}_{\mathrm{sd}}$ and $\mathbf{y}_{\text {rd }}$. To this aim, an IDMA receiver is used to separate the signals of the $q$ sources, as described above. Also, since extra parity is provided by the relay, an iterative exchange of information is also performed between decoders $\mathcal{C}_{i}^{-1}$ and the decoder $C_{\mathrm{I}}^{-1}$ of the relay, as described in Section IV-A. We define a global iteration an iteration between the IDMA receiver and decoder $C_{\mathrm{I}}^{-1}$, and a local iteration an iteration within the IDMA receiver (as described above). Here, we consider a single local iteration for each global iteration. The structure of the distributed SCC receiver with multiuser detection at the destination node is depicted in Fig. 6. 


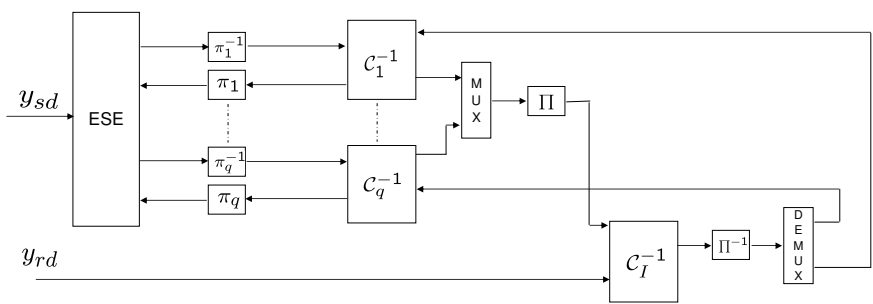

Fig. 6. IDMA receiver at the destination node.

TABLE II

MINIMUM VALUES OF $\gamma_{\text {Sd }}^{\mathrm{b}}$ ACHIEVED OVER FAST FADING CHANNEL

\begin{tabular}{lcccc}
\hline & $\gamma_{\min , \mathrm{eq}}^{\mathrm{b}}$ & $\theta_{\mathrm{s}}^{\text {opt }}$ & $\theta_{\mathrm{r}}^{\text {opt }}$ & $\gamma_{\min , \mathrm{opt}}^{\mathrm{b}}$ \\
\hline \hline$q=2, m=2$ & $-2.6875 \mathrm{~dB}$ & 0.159939 & 0.840061 & $-3.942565 \mathrm{~dB}$ \\
$q=4, m=4$ & $-2.7649 \mathrm{~dB}$ & 0.114238 & 0.885762 & $-4.11539 \mathrm{~dB}$ \\
$q=8, m=8$ & $-2.8062 \mathrm{~dB}$ & 0.09148 & 0.90852 & $-4.18918 \mathrm{~dB}$ \\
$q=12, m=8$ & $-2.09352 \mathrm{~dB}$ & 0.107409 & 0.892591 & $-3.275356 \mathrm{~dB}$ \\
$q=16, m=8$ & $-1.3928 \mathrm{~dB}$ & 0.12000 & 0.88000 & $-2.246815 \mathrm{~dB}$ \\
\hline
\end{tabular}

\section{A. Information theoretic limits}

Let $k_{i}, n_{i}^{\mathrm{sp}}, n_{\mathrm{r}}$ and $N^{\prime}$ be as defined above. We define the rate for source $\mathrm{s}_{i}$ as $R_{i}^{\prime}=k_{i} / N^{\prime}$ (in bits per channel use). The information of the $q$ sources $\mathrm{s}_{1}, \ldots, \mathrm{s}_{q}$ can be decoded reliably at the destination if the following inequalities hold

$$
\begin{aligned}
& q k_{i} \leq n_{i}^{\mathrm{sp}} C_{\mathrm{MUD}}\left(\gamma_{\mathrm{sr}}\right), \\
& q k_{i} \leq n_{i}^{\mathrm{sp}} C_{\mathrm{MUD}}\left(\gamma_{\mathrm{sd}}\right)+n_{\mathrm{r}} C\left(\gamma_{\mathrm{rd}}\right),
\end{aligned}
$$

where $C_{\text {MUD }}$ denotes the sum capacity of the MAC corresponding to the $q$ sources $\mathrm{s}_{1}, \ldots, \mathrm{s}_{q}$.

We define the time allocation parameters $\theta_{\mathrm{s}}=n_{i}^{\mathrm{sp}} / N^{\prime}$ and $\theta_{\mathrm{r}}=n_{\mathrm{r}} / N^{\prime}$ for the $q$ sources and the relay, respectively. Also, $\theta_{\mathrm{s}}+\theta_{\mathrm{r}}=1$. Dividing by $N^{\prime}$ in both sides of the inequalities (19) we obtain

$$
\begin{aligned}
& q R_{i}^{\prime} \leq \theta_{\mathrm{s}} C_{\mathrm{MUD}}\left(\gamma_{\mathrm{sr}}\right), \\
& q R_{i}^{\prime} \leq \theta_{\mathrm{s}} C_{\mathrm{MUD}}\left(\gamma_{\mathrm{sd}}\right)+\theta_{\mathrm{r}} C\left(\gamma_{\mathrm{rd}}\right) .
\end{aligned}
$$

1) Equal time allocation: We consider an equal time allocation between the $q$ sources and the relay, i.e., $\theta_{\mathrm{s}}=\theta_{\mathrm{r}}=1 / 2$. Under the assumption that all sources transmit at the same rate $\left(R_{i}^{\prime}=R_{\mathrm{s}}^{\prime} \forall i\right)$, the achievable rates $R_{i}^{\prime}, i=1, \ldots q$, are given by

$$
\begin{aligned}
R_{i}^{\prime} & =R_{\mathrm{s}}^{\prime} \\
& =\min \left\{\frac{1}{2 q} C_{\mathrm{MUD}}\left(\gamma_{\mathrm{sr}}\right), \frac{1}{2 q} C_{\mathrm{MUD}}\left(\gamma_{\mathrm{sd}}\right)+\frac{1}{2 q} C\left(\gamma_{\mathrm{rd}}\right)\right\} .
\end{aligned}
$$

The sum rate of the overall system is given by $R_{\text {eff }}=q R_{s}^{\prime}$.

In [17] the achievable sum rate for IDMA systems with nonGaussian chips was derived assuming successive interference cancelation. Here, we compute $C_{\mathrm{MUD}}$ following the approach in [17] assuming BPSK modulation. In Table II we report the minimum value of $\gamma_{\mathrm{sd}}^{\mathrm{b}}\left(\gamma^{\mathrm{b}}{ }_{\text {min,eq }}\right)$ such that a system rate $R_{\text {eff }}=\frac{q}{4 m}$ is achieved over Rayleigh fast fading channel. In the computation, we assumed $d_{\mathrm{sr}}=(1 / 4) d_{\mathrm{sd}}$ and $d_{\mathrm{rd}}=$ $(3 / 4) d_{\mathrm{sd}}$.

2) Optimal time allocation: The optimal time allocation is chosen such that the rate $R_{\mathrm{s}}^{\prime}$ is maximized.

$$
\theta_{\mathrm{s}}^{\text {opt }}=\arg \max R_{\mathrm{s}}^{\prime} \quad \text { subject to } \theta_{\mathrm{r}}=1-\theta_{\mathrm{s}} .
$$

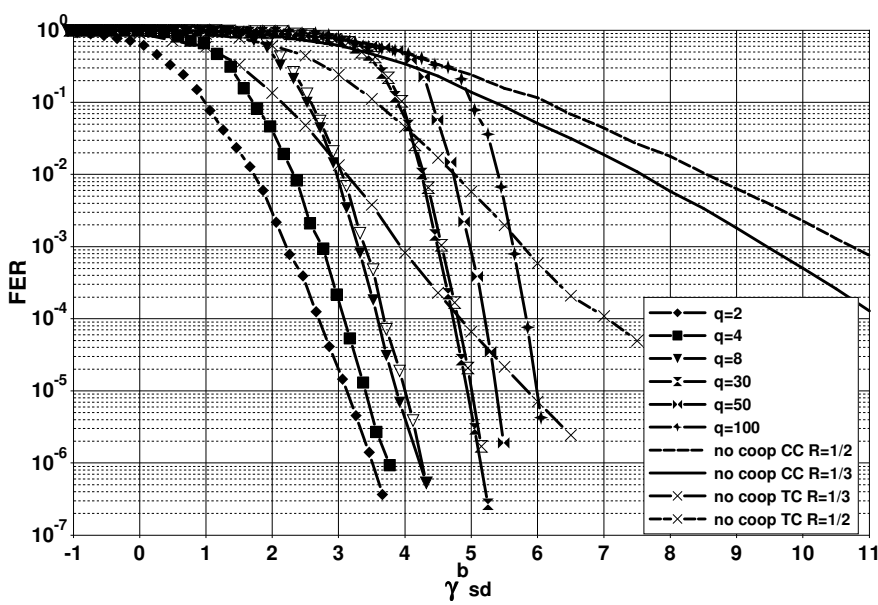

Fig. 7. FER curves for the distributed SCC of Fig. 2 using strategy A (solid markers) and B (empty markers) for $q=2,4,8,30,50$ and 100 sources and equal time allocation over Rayleigh fast fading channel. $R_{i}=R_{\mathrm{S}}=1 / 2$, $R_{\text {eff }}=\frac{q}{2(1+q)} \cdot k_{i}=k=96$ bits, 15 iterations.

We proceed in the same manner as done in Section V-B. The optimal time allocation $\theta_{s}^{\text {opt }}$ is given by

$$
\theta_{\mathrm{s}}^{\mathrm{opt}}=\frac{C\left(\gamma_{\mathrm{rd}}\right)}{C\left(\gamma_{\mathrm{rd}}\right)+C_{\mathrm{MUD}}\left(\gamma_{\mathrm{sr}}\right)-C_{\mathrm{MUD}}\left(\gamma_{\mathrm{sd}}\right)},
$$

while the achievable rates $R_{i}^{\prime}$ are given by

$$
R_{i}^{\prime}=R_{\mathrm{s}}^{\prime}=\frac{1}{q} \frac{C\left(\gamma_{\mathrm{rd}}\right) C_{\mathrm{MUD}}\left(\gamma_{\mathrm{sr}}\right)}{C\left(\gamma_{\mathrm{rd}}\right)+C_{\mathrm{MUD}}\left(\gamma_{\mathrm{sr}}\right)-C_{\mathrm{MUD}}\left(\gamma_{\mathrm{sd}}\right)} .
$$

The rate of the overall system (sum rate) can be written as

$$
R_{\mathrm{eff}}=q R_{s}^{\prime}=\frac{C\left(\gamma_{\mathrm{rd}}\right) C_{\mathrm{MUD}}\left(\gamma_{\mathrm{sr}}\right)}{C\left(\gamma_{\mathrm{rd}}\right)+C_{\mathrm{MUD}}\left(\gamma_{\mathrm{sr}}\right)-C_{\mathrm{MUD}}\left(\gamma_{\mathrm{sd}}\right)} .
$$

In Table II we report the optimal time allocation parameters $\theta_{\mathrm{s}}^{\text {opt }}$ and $\theta_{r}^{\text {opt }}$ and the minimum values of $\gamma_{\mathrm{sd}}^{\mathrm{b}}\left(\gamma_{\text {min,opt }}^{\mathrm{b}}\right)$ such that a system rate $R_{\text {eff }}=\frac{q}{4 m}$ is achieved over Rayleigh fast fading channel for different values of $q$ and $m$. An improvement of $0.8-1.4 \mathrm{~dB}$ with respect to equal time allocation is observed.

\section{Simulation Results}

For the results in this section we assume for simplicity that each source uses the same encoder $\mathcal{C}_{i}=\mathcal{C}$. We consider the $R=1 / 2$ encoders defined in Section III-C for strategies $\mathrm{A}$ and $\mathrm{B}$, punctured to higher rates whenever necessary. The extension of the proposed scheme to other constituent encoders at the sources, e.g. block codes or turbo codes, is straightforward. An S-random interleaver $\Pi$ [23] and a maximum of fifteen decoding iterations are assumed. We also assume that $d_{\mathrm{sr}}=(1 / 4) d_{\mathrm{sd}}$ and $d_{\mathrm{rd}}=(3 / 4) d_{\mathrm{sd}}$, as before. In all simulations the block length is $k_{i}=k=96$ bits and the overall system code rate is $R_{\mathrm{eff}}=\frac{q}{2(1+q)}$, unless otherwise stated.

In Fig. 7 we give frame error rate (FER) results for strategies $\mathrm{A}$ and $\mathrm{B}$ with equal time allocation for several numbers of users over a Rayleigh fast fading channel as a function of $\gamma_{\mathrm{sd}}^{\mathrm{b}}$ (in $\mathrm{dB}$ ). We recall that the amount of information transmitted by the relay can be controlled by parameters $J$ and $\rho_{p}$ for 


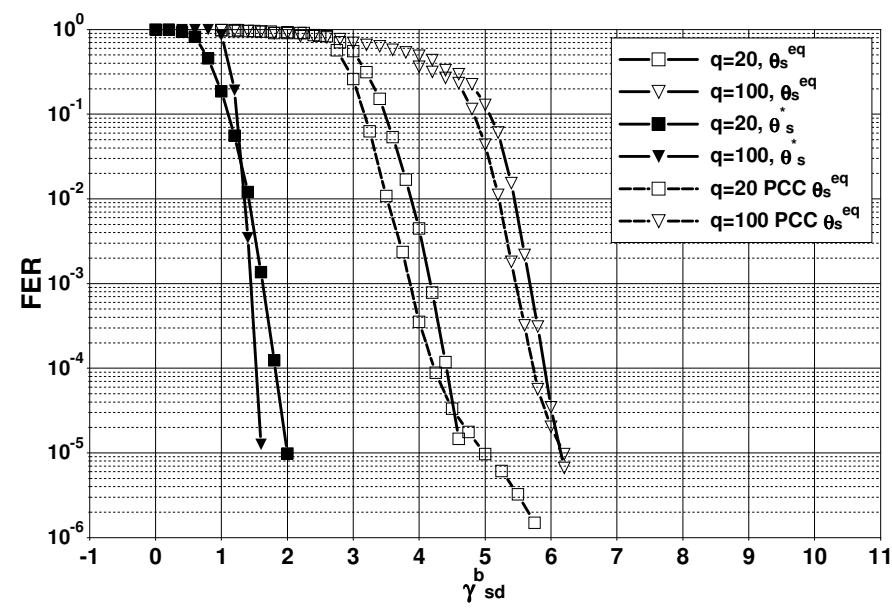

Fig. 8. FER curves for the distributed SCC of Fig. 2 using strategy B for $q=20$ and 100 sources over Rayleigh fast fading channel, for optimal (solid markers) and equal (empty markers) time allocation. $R_{\text {eff }}=\frac{q}{2(1+q)}$. $k_{i}=k=96$ bits, 15 iterations. The dashed curves correspond to the PCC for $q=20$ and 100 .

strategy $\mathrm{A}$ and $\mathrm{B}$, respectively. We consider $R_{i}=R_{\mathrm{s}}=1 / 2$. Thus, to achieve $R_{\mathrm{eff}}=\frac{q}{2(1+q)}, J=q$ and $\rho_{p}=1 / q$ (see Section III). While the block length $k$ is very short the overall distributed turbo-like code turns out to be very powerful. Very low error rates are achieved for all values of $q$. Both strategies show similar performance. Note that the curves shift to the right with increasing number of sources, since the effective code rate is higher. Note also that the curves get closer to the predicted convergence thresholds for increasing values of $q$. This result was expected, since the interleaver length (and therefore the block length of the overall SCC) increases with the number of sources. Clearly, performance will break down if a very large number of sources is considered.

For comparison purposes, we also consider the noncooperation scenario. For fair comparison, we plot two curves, assuming that each source transmits with a rate $1 / 3$ (which corresponds to the effective rate for two sources) and with rate $1 / 2$ (the effective rate tends to $R_{\text {eff }}=1 / 2$ for increasing $q$ ). We consider the use of convolutional codes (CCs) and turbo codes (TCs) at the sources. The proposed distributed turbolike code shows a significant gain with respect to the noncooperation scenario where CCs are used at each source. It also outperforms the non-cooperation scheme where a turbo code is used at the sources even for a very large number of sources $(q=100)$. Due to the short block length, the noncooperation curve using TCs at the sources shows a very high error floor. In contrast, the proposed distributed SCC is able to attain much lower error rates for the same block length, since the overall distributed (over the sources and the relay) code is very powerful.

In Fig. 8 we compare equal time allocation and optimal time allocation according to $\theta_{\mathrm{s}}^{*}$ and $\theta_{\mathrm{r}}^{*}$ (see Section V-B) for strategy $\mathrm{B}$ for $q=20$ and $q=100$ sources. The overall system rate is $R_{\text {eff }}=\frac{q}{2(1+q)}$ for both cases. For optimal time allocation, this corresponds to $R_{i}=R_{\mathrm{S}}=0.8, R_{\mathrm{I}}=1.46$ and $R_{i}=R_{\mathrm{S}}=0.8, R_{\mathrm{I}}=1.63$ for $q=20$ and $q=100$, respectively. An improvement of $\sim 2 \mathrm{~dB}$ and $\sim 4 \mathrm{~dB}$ is

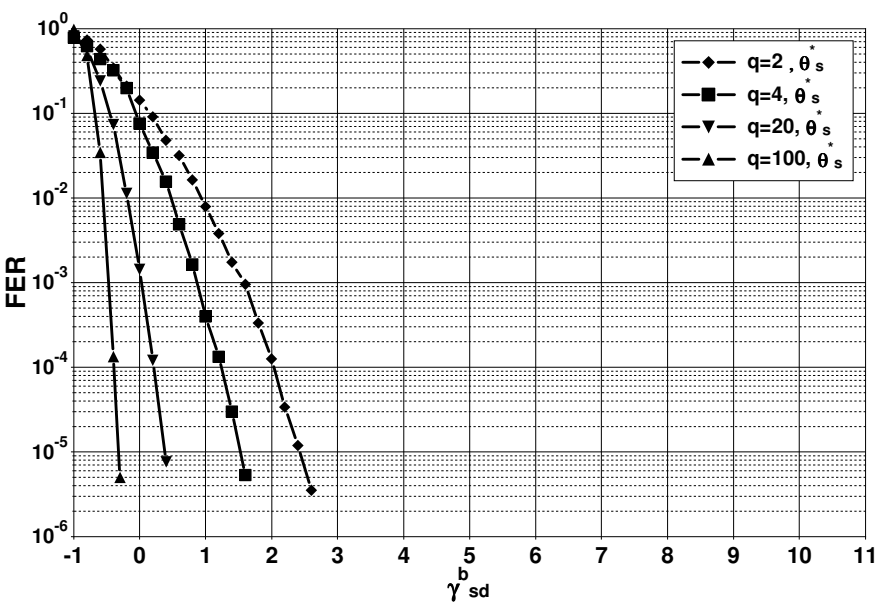

Fig. 9. FER curves for the distributed SCC of Fig. 2 using strategy B for $q=2,4,20$ and 100 sources over Rayleigh fast fading channel for a fixed rate $R_{\text {eff }}=1 / 3$ and optimal time allocation. $k_{i}=k=96$ bits, 15 iterations.

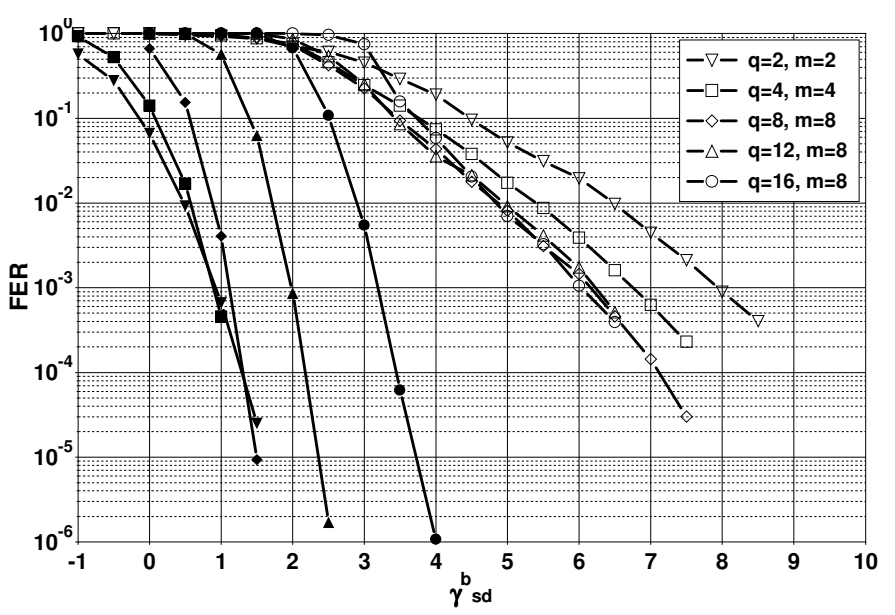

Fig. 10. FER curves, non-cooperation scheme (empty markers), cooperation scheme (filled markers), with $q=2,4,8,12$ and 16 sources, over fast fading channel. $R_{i}=R_{\mathrm{S}}=1 / 2, R_{\mathrm{eff}}=\frac{q}{4 m} \cdot k_{i}=k=96$ bits, 30 iterations.

achieved by optimal time allocation with respect to equal time allocation for $q=20$ and $q=100$, respectively. Note that the curves for $q=100$ and $q=20$ cross each other. While the curve for $q=100$ converges a bit later (see Table I) it decays more rapidly due to a larger inverleaver size. In Fig. 8, we also plot the FER curves for the distributed coding scheme working as a PCC (i.e., the relay estimates an encodes $\mathbf{u}_{i}$ instead of $\mathbf{x}_{i}$ ). A slight gain is observed in the waterfall region with respect to the distributed SCC. However, the distributed PCC suffers from an error floor between FER $10^{-4}$ and $10^{-5}$, due to its poorer minimum distance.

Fig. 9 depicts the FER curves of the proposed distributed SCC (strategy B) with constant $R_{\text {eff }}=1 / 3$ and time allocation optimized by means of EXIT charts as explained in Section VI, for $q=2,4,20$ and 100. It is shown that for a given $R_{\text {eff }}$ increasing $q$ improves performance. This is due to the fact that packing together more users a stronger (larger interleaver) overall code is obtained.

Finally, in Fig. 10 we give FER curves for the multi-source scenario with non-orthogonal channels for $q=2,4,8,12$ and 
16 sources and Rayleigh fast fading. Strategy B is considered at the relay and equal time allocation is assumed. Furthermore, $R_{i}=R_{\mathrm{s}}=1 / 2$, thereby $R_{\text {eff }}=\frac{q}{4 m}$. Also, a different random interleaver $\pi_{i}$ is used for each user, and different spreading factors $m$ are considered for different values of $q$. A significant gain is observed with respect to the non-cooperation system.

For multiuser communications, orthogonal and non orthogonal techniques can be used. With respect to TDMA, it was demonstrated in [24] that IDMA achieves higher spectral and power efficiency in fading environments. In [24], the authors provided a comparison between orthogonal (TDMA, FDMA) and non orthogonal approaches (CDMA, IDMA). For applications which are delay insensitive, e.g. e-mail, orthogonal schemes are advantageous while for delay sensitive services, e.g. speech and video non orthogonal schemes are advantageous. The comparison provided in [24] remains valid for our system.

\section{CONClusions}

We proposed a distributed turbo-like code for a relay wireless network where multiple sources transmit to a destination with the help of a common relay, which is closer to the sources than to the destination. The relay uses a decode-and-forward strategy and operates in half-duplex mode. We considered both orthogonal and non-orthogonal channels. For the latter, IDMA is used for multiuser detection.

The proposed scheme can be regarded as a distributed SCC, where the relay takes the role of the inner encoder: the relay decodes the transmitted codewords from all sources and properly combines and re-encodes them to generate some extra redundancy. At the receiver side, a decoding strategy that resembles decoding of SCCs can be used. The proposed distributed SCC achieves very low error rates in both orthogonal and non-orthogonal scenarios even for a large number of sources and very short block lengths and a significant gain with respect to the non-cooperation case. Furthermore, it is very flexible; the amount of redundancy transmitted by the relay can be easily adjusted according to the number of sources, error rate requirements, overall system rate, optimal time allocation, and/or power constraints by a simple tuning parameter (parameter $J$ and parameter $\rho_{p}$ for strategy A and $\mathrm{B}$, respectively).

\section{APPENDIX}

The optimization of the time allocation is solved following the approach in [21]. Under the assumption of a symmetric system, i.e., $\theta_{1}=\theta_{2}, R_{1}^{\prime}=R_{2}^{\prime}$, we can rewrite the conditions in (5) as follows

$$
\begin{aligned}
& R_{1}^{\prime} \leq \theta_{1} C\left(\gamma_{\mathrm{sr}}\right), \\
& R_{1}^{\prime} \leq \theta_{1} C\left(\gamma_{\mathrm{sd}}\right)+\left(1-2 \theta_{1}\right) C\left(\gamma_{\mathrm{rd}}\right), \\
& R_{1}^{\prime} \leq \theta_{1} C\left(\gamma_{\mathrm{sd}}\right)+\frac{1}{2}\left(1-2 \theta_{1}\right) C\left(\gamma_{\mathrm{rd}}\right) .
\end{aligned}
$$

The optimization is done in two steps. First, the optimal time allocation parameter $\theta_{1}^{\text {opt }}$ is determined taking into account conditions (26) and (28). Then, with this time allocation parameter, condition (27) is shown to be not relevant for
$C\left(\gamma_{\mathrm{sr}}\right)>C\left(\gamma_{\mathrm{sd}}\right)$. We will first determine the optimal value $\theta_{1}^{\mathrm{opt}} . \theta_{1} C\left(\gamma_{\mathrm{sr}}\right)$ is monotone increasing with $\theta_{1}$ and with $C\left(\gamma_{\mathrm{sd}}\right)<C\left(\gamma_{\mathrm{rd}}\right)$ we have that $\theta_{1} C\left(\gamma_{\mathrm{sd}}\right)+\frac{1}{2}\left(1-2 \theta_{1}\right) C\left(\gamma_{\mathrm{rd}}\right)$ is monotone decreasing with $\theta_{1}$. The optimal value $\theta_{1}^{\text {opt }}$ is found at the cross point of $\theta_{1} C\left(\gamma_{\mathrm{sr}}\right)$ and $\theta_{1} C\left(\gamma_{\mathrm{sd}}\right)+\frac{1}{2}(1-$ $\left.2 \theta_{1}\right) C\left(\gamma_{\mathrm{rd}}\right)$. Solving the following equation gives the optimal parameter $\theta_{1}^{\text {opt }}$ given in (15).

$$
\theta_{1}^{\mathrm{opt}} C\left(\gamma_{\mathrm{sr}}\right)=\theta_{1}^{\mathrm{opt}} C\left(\gamma_{\mathrm{sd}}\right)+\frac{1}{2}\left(1-2 \theta_{1}^{\mathrm{opt}}\right) C\left(\gamma_{\mathrm{rd}}\right)
$$

Now, we will show that condition (27) is not relevant for $C\left(\gamma_{\mathrm{sr}}\right)>C\left(\gamma_{\mathrm{sd}}\right)$. From (29), we have that

$$
\begin{aligned}
\theta_{1}^{\mathrm{opt}} C\left(\gamma_{\mathrm{sd}}\right)+\left(1-2 \theta_{1}^{\mathrm{opt}}\right) C\left(\gamma_{\mathrm{rd}}\right) & =2 \theta_{1}^{\mathrm{opt}} C\left(\gamma_{\mathrm{sr}}\right)-\theta_{1}^{\mathrm{opt}} C\left(\gamma_{\mathrm{sd}}\right) \\
& =\theta_{1}^{\mathrm{opt}} C\left(\gamma_{\mathrm{sr}}\right)\left(2-\frac{C\left(\gamma_{\mathrm{sd}}\right)}{C\left(\gamma_{\mathrm{sr}}\right)}\right)
\end{aligned}
$$

which is larger than $\theta_{1}^{\mathrm{opt}} C\left(\gamma_{\mathrm{sr}}\right)$ for $C\left(\gamma_{\mathrm{sd}}\right) \leq \theta_{1}^{\mathrm{opt}} C\left(\gamma_{\mathrm{sr}}\right)$.

\section{REFERENCES}

[1] E. C. van der Meulen, "Three-terminal communication channels," $A d v$. Appl. Prob., vol. 3, no. 1, pp. 120-154, Jun. 1971

[2] T. Cover and A. E. Gamal, "Capacity theorems for the relay channel," IEEE Trans. Inf. Theory, vol. 25, no. 5, pp. 572-584, Sep. 1979.

[3] M. Valenti and B. Zhao, "Distributed turbo codes: towards the capacity of the relay channel," in Proc. IEEE Vehicular Technology Conference (VTC), Oct. 2003, pp. 322-326.

[4] Z. Zheng and T. Duman, "Capacity-approaching turbo coding and iterative decoding for relay channels," IEEE Trans. Inf. Theory, vol. 53, pp. 1895 - 1905, Nov. 2005.

[5] C. Hausl, F. Schrechenbach, I. Oikonomidis, and G. Bauch, "Iterative network and channel decoding on a Tanner graph," in Proc. 39th Annual Allerton Conf. on Commun., Control, and Computing, Sep. 2005.

[6] S. Yang and R. Koetter, "Network coding over a noisy relay : a belief propagation approach," in Proc. IEEE Int. Symp. Inf. Theory (ISIT), Jun. 2007, pp. 801-804.

[7] M. Dohler and Y. Li, Cooperative Communications: Hardware, Channel and PHY. Wiley \& Sons, 2010.

[8] R. Pyndiah, F. Guilloud, and K. Amis, "Multiple source cooperative coding using block turbo codes," in Proc. 6th International symposium on turbo codes \& iterative information processing, Sep. 2010.

[9] M. D. Yacoub, Foundations of Mobile Radio Engineering. CRC Press, 1993.

[10] T. Rappaport, Wireless Communications. McGraw Hill International Editions series, 1999.

[11] H. Holma and A. Toskala, WCDMA for UMTS. Wiley, Inc., 2001.

[12] R. Lupas and S. Verdu, "Linear multiuser detector for synchronous codedivision multiple-access channels," IEEE Trans. Inf. Theory, vol. 35, no. 1, pp. 123-136, Jan. 1989.

[13] L. Ping, L. Liu, K. Y. Wu, and W. K. Leung, "Interleave-division multiple-access (IDMA) communications," in Proc. 3rd International Symposium on Turbo Codes and Related Topics, Sep. 2003, pp. 173180.

[14] X. Wang and H. Poor, "Iterative (turbo) soft interference cancellation and decoding for coded CDMA," IEEE Trans. Commun., vol. 47, no. 7, pp. 1046-1061, Jul. 1999.

[15] L. Ping, L. Liu, K. Y. Wu, and W. K. Leung, "A simple approach to near optimal multiuser detection: Interleave-division multiple-access," in Proc. IEEE WCNC 2003-Wireless Communications and Networking Conf., Mar. 2003, pp. 391-396.

[16] —, "Interleave-division multiple-access," IEEE Trans. Wireless Commun., vol. 5, no. 4, pp. 938-947, Apr. 2006.

[17] P. Hoeher and H. Schoeneich, "Interleave-division multiple access from a multiuser theory point of view," in Proc. 4th International Symposium on Turbo Codes and Related Topics, Apr. 2006.

[18] S. Benedetto, D. Divsalar, G. Montorsi, and F. Pollara, "Serial concatenation of interleaved codes: Performance analysis, design and iterative decoding," IEEE Trans. Inform. Theory, vol. 44, no. 3, pp. 909-926, May 1998. 
[19] K. M. Chugg, P. Thiennviboon, G. D. Dimou, P. Gray, and J. Melzer, "A new class of turbo-like codes with universally good performance and high-speed decoding," in Proc. IEEE Military Communications Conference (MILCOM), Oct. 2005, pp. 3117-3126.

[20] A. Graell i Amat, G. Montorsi, and F. Vatta, "Design and performance analysis of a new class of rate compatible serially concatenated convolutional codes," IEEE Trans. Commun., vol. 57, no. 8, pp. 2280-2289, Aug. 2009.

[21] C. Hausl, Joint Network-Channel Coding for Wireless Relay Networks. $\mathrm{PhD}$ thesis, 2008.

[22] S. ten Brink, "Convergence behaviour of iteratively decoded parallel concatenated codes," IEEE Trans. Commun., vol. 49, no. 10, pp. 1727 1737, Oct. 2001.

[23] D. Divsalar and F. Pollara, "Turbo codes for PCS applications," in Proc. IEEE Int. Conf. Communications, Jun. 1995, pp. 54-59.

[24] P. Wang, J. Xio, and L. Ping, "Comparison of orthogonal and nonorthogonal approaches to future wireless cellular systems," IEEE Vehic. Tech. Magazine, vol. 1, no. 3, pp. 4-11, Sep. 2006.

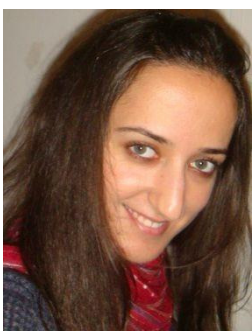

Roua Youssef (S'09) was born in Beirut, Lebanon, in 1984. In 2007, she received her diploma in Telecommunications Engineering from the Lebanese University of Beirut, Lebanon, and the M.Sc. degree in Electrical Engineering from TELECOM Bretagne (former ENST Bretagne), Brest, France. She is currently working toward her Ph.D. degree at the Department of Electronics of TELECOM Bretagne. Her research interests are in the area of cooperative communications and distributed coding for wireless networks.

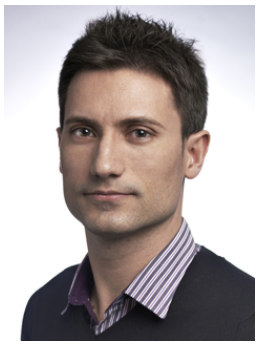

Alexandre Graell i Amat (S'01 - M'05 - SM'10) was born in Barcelona, Catalonia, Spain, on January 28,1976 . He received the M.Sc. degree in Telecommunications Engineering from the Universitat Politècnica de Catalunya, Barcelona, Catalonia, Spain, in 2001, and the M.Sc. and the Ph.D. degrees in Electrical Engineering from the Politecnico di Torino, Turin, Italy, in 2000 and 2004, respectively. From September 2001 to April 2002, he was a Visiting Scholar at the Center for Magnetic Recording Research, University of California at San Diego, La Jolla, CA. From September 2002 to May 2003, he held a visiting appointment at Universitat Pompeu Fabra, and at the Telecommunications Technological Center of Catalonia, both in Barcelona. During 2001- 2004, he also held a part-time appointment at STMicroelectronics Data Storage Division, Milan, Italy, as consultant on coding for magnetic recording channels. From March 2004 to December 2005, he was a Visiting Professor at Universitat Pompeu Fabra. Since January 2006 he has been with the Department of Electronics of TELECOM Bretagne (former ENST Bretagne), Brest, France, where he is currently an Associate Professor. His research interests are in the area of Communication Theory and Information Theory, and include errorcontrol coding, iterative decoding algorithms, cooperative communications, and MIMO systems.

Dr. Graell i Amat is currently an Editor of the IEEE COMMUNICATIONS LETTERS. He received the post-doctoral Juan de la Cierva Fellowship of the Spanish Ministry of Education and Science, and the Marie Curie IntraEuropean Fellowship of the European Commission. 